\title{
Dissociable effects of noradrenaline, dopamine, and serotonin uptake blockade on stop task performance in rats
}

\author{
Andrea Bari • Dawn M. Eagle • Adam C. Mar • \\ Emma S. J. Robinson • Trevor W. Robbins
}

Received: 14 December 2008 / Accepted: 2 April 2009/Published online: 30 April 2009

(C) Springer-Verlag 2009

\begin{abstract}
Rationale The stop-signal paradigm measures the ability to stop a motor response after its execution has been initiated. Impairments in inhibiting inappropriate behavior and prolonged stop-signal reaction times (SSRTs) are characteristic of several psychiatric disorders, most notably attention deficit/hyperactivity disorder. While there is relative consensus regarding the anatomical substrates of behavioral inhibition, the neurochemical imbalance responsible for the deficits in stopping displayed by impulsive individuals is still a matter of debate.

Objective The aim of this study was to investigate the effects of manipulating brain monoamine levels on stop task parameters.

Methods Lister-hooded rats were trained on the rodent version of the stop-signal task and administered different monoamine transporter inhibitors: citalopram, which selectively blocks the serotonin transporter; atomoxetine, which selectively blocks the noradrenaline transporter; and GBR12909 , which selectively blocks the dopamine transporter (DAT), and the alpha-2 adrenergic agonist guanfacine.

Results Atomoxetine speeded SSRT and increased accuracy for go-trials. Citalopram slowed go reaction time and
\end{abstract}

\footnotetext{
A. Bari • D. M. Eagle $(\bowtie) \cdot$ A. C. Mar $\cdot$ T. W. Robbins Department of Experimental Psychology and Behavioural and Clinical Neuroscience Institute,

University of Cambridge,

Downing Street,

Cambridge, CB2 3EB, UK

e-mail: d.eagle@psychol.cam.ac.uk

E. S. J. Robinson

Department of Physiology and Pharmacology,

University of Bristol, School of Medical Sciences,

University Walk,

Bristol, BS8 1TD, UK
}

decreased go accuracy at the highest dose $(1 \mathrm{mg} / \mathrm{kg})$. GBR-12909 speeded go reaction time and impaired both go and stop accuracy. Guanfacine negatively modulated all principal stop and go measures at the highest dose used $(0.3 \mathrm{mg} / \mathrm{kg})$.

Conclusions The results suggest that atomoxetine exerts its beneficial effects on SSRT via its action on noradrenaline re-uptake, as the specific DAT blocker GBR-12909 and serotonin reuptake blockade had only minor effects on SSRT. The speeding of the go reaction time by dopamine reuptake blockade is consistent with the hypothesis that the hypothetical stop and go processes are modulated by distinct monoaminergic systems.

Keywords Stop-signal task · Response inhibition · SSRT . Dopamine · Noradrenaline · Serotonin · GBR-12909 .

Atomoxetine $\cdot$ Citalopram $\cdot$ Guanfacine

\section{Introduction}

Behavioral inhibition is one of the most studied constructs in psychopathology and is known to be dysfunctional in a wide range of psychiatric conditions characterized by impulsive behavior such as personality disorders, mania, substance abuse and attention deficit/hyperactivity disorder (ADHD) (Barkley 1997; Groman et al. 2007; Moeller et al. 2001). The stop-signal paradigm (Logan 1994; Logan et al. 1984) measures a specific kind of executive inhibition often referred to as motor or behavioral inhibition and is sufficiently sensitive to differentiate impulsive individuals from the normal population (Oosterlaan et al. 1998; Rubia et al. 1998). Investigation of the neural and neurochemical mechanisms necessary for inhibitory control, however, requires the use of a suitable analog of the stop-signal task 
in experimental animals (Eagle et al. 2007, 2008; Eagle and Robbins 2003).

Of the main neuromodulatory systems of the brain, serotonin (5-HT), dopamine (DA), and noradrenaline (NA) appear to play crucial roles in different forms of behavioral inhibition (Robbins 2007). A family of proteins known as cell membrane monoamine transporters, which are targets for many therapeutic compounds and drugs of abuse, enable the regulation of extracellular levels of monoamines (Iversen 2006; Masson et al. 1999). Indeed, the most widely used drugs in the pharmacotherapy of ADHD (i.e., amphetamine, methylphenidate, and recently, atomoxetine) act on membrane transporters to inhibit the reuptake of catecholamines (Heal et al. 2008).

While functional neuroimaging studies point to corticostriatal circuitry and, more specifically, to the right inferior frontal gyrus and the striatum as playing an important role in the mediation of stopping behavior (Aron et al. 2003; Robbins 2007; Rubia 2002; Semrud-Clikeman et al. 2000; Vaidya et al. 1998), the neurochemical substrates responsible for the modulation of the cerebral areas involved in response inhibition as measured by the stop-signal task have yet to be precisely defined.

We have recently shown that atomoxetine, a selective noradrenaline reuptake inhibitor (SNRI), has dosedependent, beneficial effects on different forms of impulsivity in the rat (Robinson et al. 2008). A key question in the psychopharmacology of impulsive behavior is whether these effects are mainly mediated by dopamine, noradrenaline, or both. In fact, atomoxetine, despite its high selectivity for the noradrenaline transporter (NET), increases in vivo extracellular levels of both catecholamines in prefrontal cortex (PFC; Bymaster et al. 2002), in addition to acetylcholine and histamine (Horner et al. 2007; Tzavara et al. 2006). Atomoxetine shares some of these properties with other drugs used for the treatment of ADHD such as amphetamine and methylphenidate (Horner et al. 2007; Tanda et al. 1997; Tzavara et al. 2006), but it lacks significant dopaminergic modulation in nucleus accumbens (NAc) and striatum (STR) (Bymaster et al. 2002), which is a characteristic of stimulant drugs. GBR12909 , instead, is a highly selective inhibitor of the dopamine transporter (DAT) with little affinity for the noradrenaline and serotonin (SERT) transporters (Rothman et al. 2001).

In order to investigate the consequences of independently blocking 5-HT, NE, and DA transporters on stop task variables, we tested, in a series of discrete experiments, the effects of systemic administration of citalopram, atomoxetine, and GBR-12909, respectively. To further explore the effect of modulating noradrenergic tone, we also used the selective alpha-2 adrenergic agonist guanfacine (Easton et al. 2006), which has been shown to ameliorate ADHD symptoms in humans and other animals (Sagvolden 2006; Scahill et al. 2001; Taylor and Russo 2001) and to regulate DA and NA neurotransmission in PFC (Devoto et al. 2004). Comparisons of these drugs may (a) illuminate the precise mode of action of atomoxetine in its effect on stop-signal reaction time (SSRT) and (b) test the hypothesis that go reaction time (GoRT) is independently modulated by dopamine (Eagle et al. 2007).

An important additional aim of these studies was to validate the rodent version of the stop task against results acquired with human subjects in our laboratory (Chamberlain et al. 2006; Clark et al. 2005; Müller et al. 2005) and to test the effect of a broader range of drug doses which is usually limited, for obvious ethical reasons, in experiments employing human subjects.

\section{Materials and methods}

\section{Subjects}

After excluding those rats that were not performing according to pre-determined criteria ( $n 33$; see below), a total of 87 male Lister-hooded rats (Charles River, UK) was used in four different experiments: 26 animals took part in experiment 1,24 in experiment 2,12 in experiment 3, and 25 in experiment 4 . For each experiment, their weights ranged from 350 to $470 \mathrm{~g}$, and the mean age was approximately 4 months at the start of testing. All subjects were housed in groups of four, under a reversed 12:12-h lightdark cycle (lights off at 07:30) and were tested during the dark phase of this cycle. In all four experiments, rats were approximately $85 \%$ of the weights of free-feeding individuals. Weight gain was restricted by feeding with a total of $15-20 \mathrm{~g}$ of food per day (reinforcer pellets during the task plus standard laboratory chow). Food restriction started at least 1 week before the beginning of training.

Water was freely available except during test sessions. All experiments were conducted in accordance with the United Kingdom Animals (Scientific Procedures) Act, 1986.

\section{Apparatus}

All sessions were performed in six operant conditioning chambers (Med Associates, VT, USA). Each experimental chamber had two retractable levers positioned $70 \mathrm{~mm}$ above the floor and $80 \mathrm{~mm}$ to either side of a central food well. A pellet dispenser delivered 45-mg Noyes Formula P pellets (Sandown Scientific, Middlesex, UK) into the food well, where head entries were monitored with an infrared detector. A houselight in the roof of the chamber remained on throughout the session with the exception of time-out 
(TO, i.e., $5 \mathrm{~s}$ of darkness) periods. A central light, positioned above the food well, signaled reinforcement delivery and/or that a nose-poke was required to start a new trial. Lights above the left and right levers signaled presentation of the corresponding lever. A 4,500-Hz Sonalert tone generator (Med Associates) was mounted high on the wall opposite to the levers and food well. Control of the experimental chambers and on-line data collection were conducted using the Whisker control system (Cardinal and Aitken 2001) and a custom software written in $\mathrm{C}++$ by DME and JMC England.

\section{Stop task}

All rats were trained to perform the stop task following a training program that has been previously described in detail (Eagle and Robbins 2003). During training and between-drug baseline sessions, rats received one 20-min session per day, with a maximum of 200 trials. In all sessions, trials were initiated with a nose-poke to the food well, after which the left lever and left light were presented. A press on the left lever resulted in the right lever and right light being presented, and the left lever and left light were withdrawn/extinguished. Rats were trained to perform a rapid operant response from left lever to right lever - the go response. Response speed was maintained by limiting the time for which the right lever was presented - the limited hold (LH; ranging from 0.8 to $1.5 \mathrm{~s}$ in experiments 1 and 4 and $1.1 \mathrm{~s}$ for all the animals in experiments 2 and 3, maintained at a constant value for each rat throughout the study). During go-trials, rats were rewarded with a pellet delivered to the central food well for pressing the right lever, but received a TO if they failed to press the right lever within the LH period. On $20 \%$ of the trials, the stoptrials, a tone $(40 \mathrm{~ms}, 4,500 \mathrm{~Hz}, \sim 80 \mathrm{~dB})$ was presented at a predetermined time between the left and right lever presses. Stop-trials were presented randomly within the session to discourage the rats from anticipating presentation of the stop-signal. On stop-trials, the rats were required to initiate the same operant response as on go-trials but, after hearing the stop-signal, to refrain from pressing the right lever. Rats were required to withhold from responding for the $\mathrm{LH}$ period, after which they were rewarded with a pellet. An incorrect response in a stop-trial - which was a press on the right lever-resulted in a TO. Following initial training, rats received 20-min baseline sessions, during which, the stopsignal was presented as the left lever was pressed (i.e., with no delay between the onset of the go response and the presentation of the stop-signal; zero delay-ZD). Mean gotrial reaction time (mRT) and stop-signal delays (SSDs) for each rat were obtained across three ZD sessions. Over the following five sessions, individual rat inhibition functions (IF) were generated (see Fig. 1 for a representative IF averaged across subjects: $n=26$ ), with SSDs presented in a randomized order from the following set: $\mathrm{SSD}=\mathrm{mRT}-$ $600 \mathrm{~ms}$; mRT-500 ms; mRT-400 ms; mRT-300 ms; mRT$200 \mathrm{~ms}$. Prior to drug testing, rats were removed from the study if their inhibition functions showed an increase (rather than a decrease) in stop-trial accuracy across increasingly difficult delays or if they were slowing down their GoRT waiting for the stop-signal to occur. A closer analysis of the performance of the remaining rats revealed that they were performing the task according to the assumptions of the "Race" model (see Logan 1994). In particular, the model assumes that go and stop processes are independent from each other and that the SSDs bias the race in favor of one or the other process.

On days when SSRT was calculated (SSRT sessions for drug-testing days and baselines), rats performed one session per day that was divided into $3 \times 10$-min test periods, with a maximum of 80 trials per period. Data from the first period at ZD were used to calculate $\mathrm{mRT}$ for each rat, and this $\mathrm{mRT}$ was used to set the SSDs in the subsequent two periods, presenting the stop signals at mRT $-500 \mathrm{~ms}$ and at mRT-300 ms. SSRT was estimated from the data of the last two periods. Twenty percent of trials in each test period were randomly determined to be stop-trials (16 trials per 80-trial period). During drug testing, drugs were given on Tuesdays and Fridays, with baseline sessions on Mondays and Thursdays.

\section{Drug administration}

All rats were tested for baseline performance prior to the beginning of the drug treatment. They were then divided into as many groups as the number of drug doses used in the specific experiment and matched for SSRT and mRT. All groups received the drug according to a fully

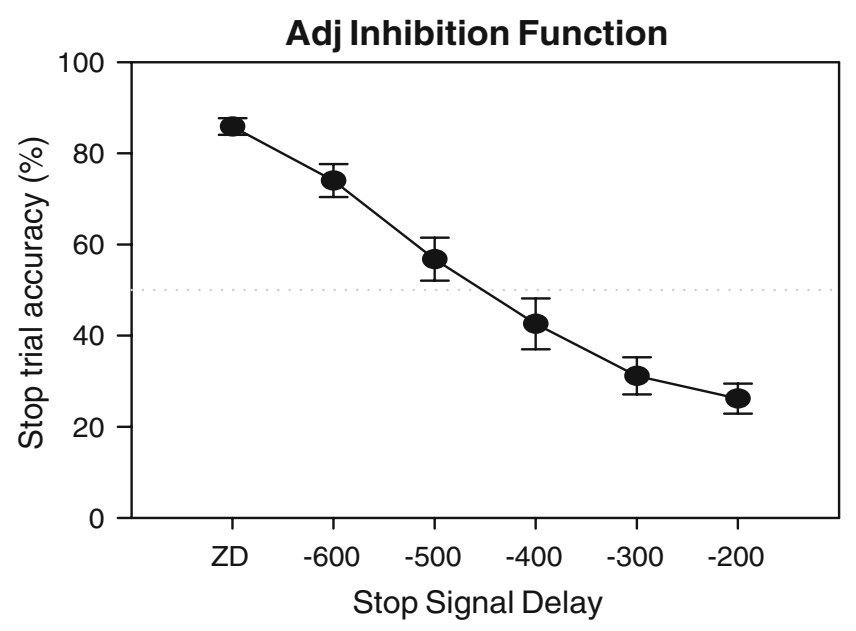

Fig. 1 Representative inhibition function obtained by plotting the stop-signal delays against the probability of successful response inhibition. $Z D$ zero delay 
randomized Latin-square design. On every test day, the drugs were freshly dissolved and administered by subcutaneous (s.c.) injections at $1 \mathrm{ml} / \mathrm{kg}$ except in experiment 3 (see below). In experiment 1, citalopram hydrobromide (four doses, 0.03, 0.1, 0.3, and $1 \mathrm{mg} / \mathrm{kg}$, plus vehicle; Tocris, Bristol, UK) was dissolved in $0.01 \mathrm{M}$ phosphate buffer saline (PBS) and administered $30 \mathrm{~min}$ before the test sessions. In experiment 2, atomoxetine (one dose, $1 \mathrm{mg} / \mathrm{kg}$, plus vehicle; Eli Lilly \& Co Ltd) was dissolved in PBS and administered $30 \mathrm{~min}$ before test sessions. In experiment 3, GBR-12909 (Tocris) was dissolved in distilled water and administered s.c. at $1 \mathrm{ml} / \mathrm{kg}$ for the first part of the experiment (four doses, 0.1, 0.3, 1, and $3 \mathrm{mg} / \mathrm{kg}$, plus vehicle), by intraperitoneal (i.p.) administration at $2 \mathrm{ml} / \mathrm{kg}$ for the second part (one dose, $5 \mathrm{mg} / \mathrm{kg}$, plus vehicle) and at $4 \mathrm{ml} / \mathrm{kg}$ i.p. for the third part (1 dose, $10 \mathrm{mg} / \mathrm{kg}$, plus vehicle). GBR-12909 was always administered $30 \mathrm{~min}$ before tests sessions. In experiment 4, guanfacine (kindly provided by Pfizer Inc., four doses, 0.01, 0.03, 0.1, and $0.3 \mathrm{mg} / \mathrm{kg}$, plus vehicle) was dissolved in vehicle containing $1 \%$ cremophor and $0.5 \%$ methylcellulose and administered $1 \mathrm{~h}$ before the test commenced. On the first day of testing a group of rats received $1 \mathrm{mg} / \mathrm{kg}$ of guanfacine, but this dose was subsequently discontinued due to excessive sedation. Drug doses and administration modalities were chosen based on published and unpublished reports showing in vivo efficacy in functional neurochemistry assays (e.g., Bymaster et al. 2002; Tanda et al. 1997)

During the time between the administration of the compound and the beginning of the task, animals were singly housed in holding cages and left undisturbed in a quiet room.

\section{Data analysis}

Data are presented for drug doses plus vehicle. Collected data were compiled in a relational database (Microsoft Access 2002) and analyzed using SPSS 12.0.1 (SPSS, Chicago, IL, USA). Graphs were plotted using SigmaPlot 8.0 (SPSS).

Behavioral data were subjected to repeated-measures analyses of variance (ANOVAs) using a general linear model. All tests of significance were performed at $\alpha=0.05$ and models were full factorial. Homogeneity of variance was verified using Levene's test. For repeated-measured analyses, Mauchly's test of sphericity was applied and the degrees of freedom corrected to more conservative values using the Huynh-Feldt epsilon $\varepsilon$ for any terms involving factors in which the sphericity assumption was violated. Corrected degrees of freedom are presented rounded to the nearest integer. Following repeated-measures analyses, pairwise comparisons were performed, with $\alpha$ adjusted using Sidak's method $\alpha^{\prime}=1-(1-\alpha)^{1 / c}$, where $c$ is the number of within-experiment analyses (Howell 1997).
For each drug, the following variables have been analyzed: SSRT, mRT, stop accuracy, and go accuracy. mRT represents the mean of reaction times (RTs) during trials in which no stop-signal was presented. Stop and go accuracy represent the proportion of successful stop and go trials, respectively, and are expressed as percentage.

SSRTs were estimated using the protocol described in Logan (1994). GoRTs were rank ordered. We selected the $n$th RT from the ranked list of GoRTs at a specific delay, where $n$ was obtained by multiplying the number of GoRTs in the distribution by the probability of responding on stop-trials at that delay. This gave an estimate of the time at which the stopping process finished, relative to the onset of the gosignal. To estimate SSRT (the time at which stopping finished relative to the stop-signal), the SSD was subtracted from this finishing time value. SSRTs from the second test period (mRT-500) and the third (mRT-300) were then averaged to obtain a single value (estimated SSRT) for each rat.

The probability of inhibiting the response on stop-trials was corrected for the presence of omission errors (trials in which the rat failed to respond for a reason unrelated to a slowing of SSRT, such as inattention or distraction) following a procedure described fully in Eagle and Robbins (2003) and is presented as "adjusted stop accuracy".

\section{Results}

Experiment 1: effects of citalopram administration

Repeated-measures analyses of variance (ANOVA) failed to reveal a main effect of citalopram doses tested on estimated SSRT (Fig. 2, upper left panel). A main effect of citalopram was found on $\operatorname{mRT}(F(4,100)=5.2, p<0.05)$. Pairwise comparisons revealed a slowing effect of the highest dose tested $(1 \mathrm{mg} / \mathrm{kg}$ ) compared to vehicle (Fig. 2, upper right panel). There were no effects of any dose on the adjusted stop accuracy (Fig. 2, lower left panel). Citalopram significantly affected go-trial accuracy (Fig. 2, lower right panel) at $1 \mathrm{mg} / \mathrm{kg},(F(4,100)=4.6, p<0.05)$; simple effects analysis revealed that, at this dose, go-trial accuracy was significantly lower compared with all other doses used.

Experiment 2: effects of a single dose of atomoxetine

Atomoxetine (1 mg/kg; Fig. 3), as previously reported in a fuller dose-response analysis (Robinson et al. 2008), significantly decreased estimated SSRT as compared to vehicle performance $(F(1,23)=6.99, p \leq 0.01)$. Atomoxetine significantly slowed the $\operatorname{mRT}(F(1,23)=19.8, p<$ $0.001)$. Go-trial accuracy was increased at the dose tested $(F(1,23)=5.65, p \leq 0.02)$ and there was a trend toward an improvement for the adjusted stop accuracy. 
Fig. 2 Citalopram had no effect on estimated SSRT (upper left) and adjusted stop-trial accuracy (lower left). mRT was significantly slower at $1 \mathrm{mg} / \mathrm{kg}$ (upper right) and the same dose significantly decreased go-trial accuracy (lower right). ${ }^{*} p<0.05$ vs. vehicle; $n=26$
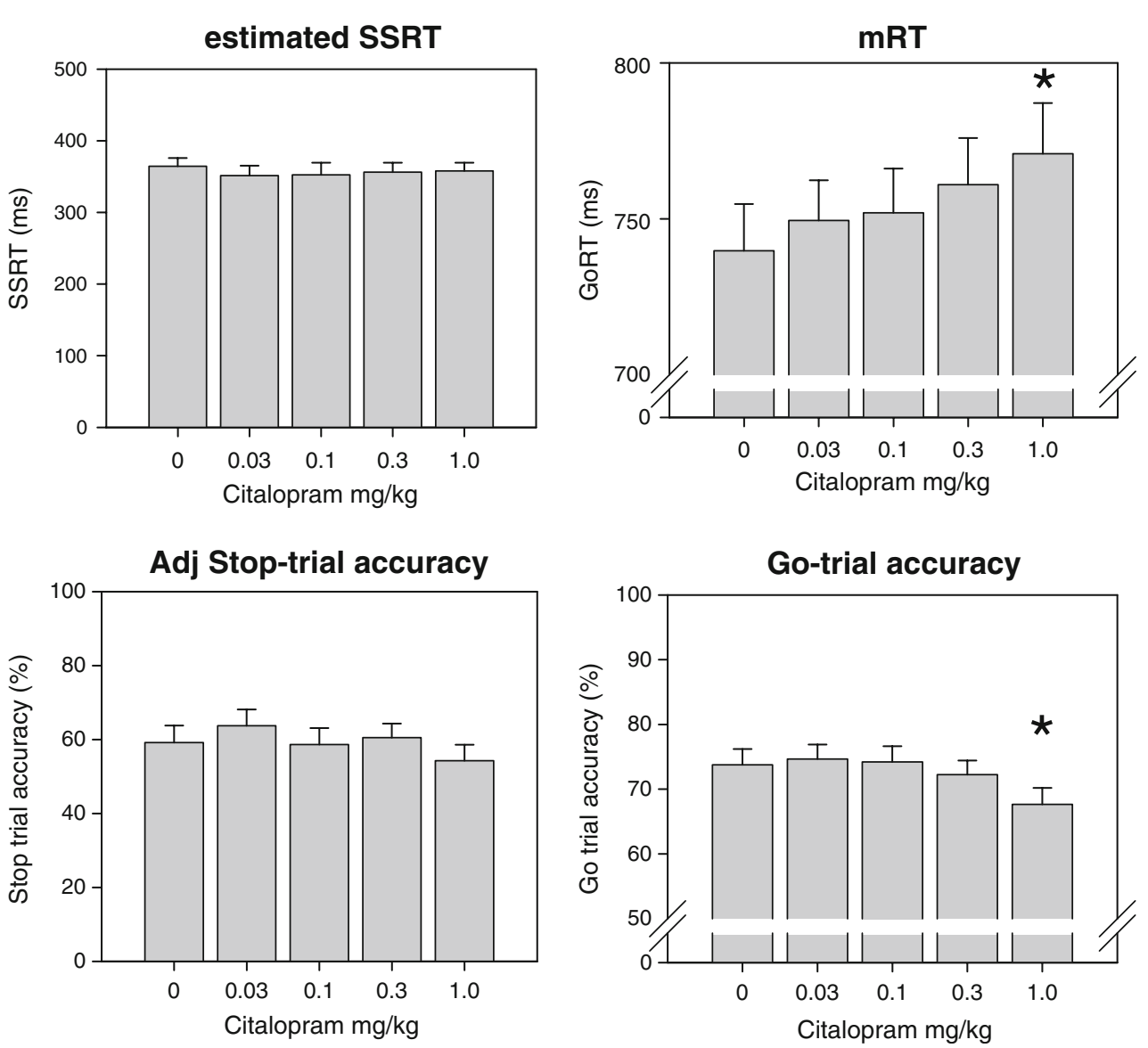

Experiment 3: effects of GBR-12909 administration

Subcutaneous administration of GBR-12909 had no significant effect on any stop task parameter with the first set of doses used (data not shown). Five milligrams per kilogram of the same drug (Fig. 4, upper panel) administered via i.p. injection speeded $\operatorname{mRT}(F(1,11)=5.527, p<0.05)$, but not
SSRT. At this dose, adjusted stop accuracy $(F(1,11)=$ 7.694, $p<0.05$ ), but not go-trial accuracy, was significantly decreased. Ten milligrams per kilogram (Fig. 4, lower panel) did not have any effect on mRT. SSRT was also not influenced by GBR-12909, and ANOVA revealed a significant decrease in both stop $(F(1,11)=5.826, p<$ $0.05)$ and go-trial $(F(1,11)=6.332, p<0.05)$ accuracy.
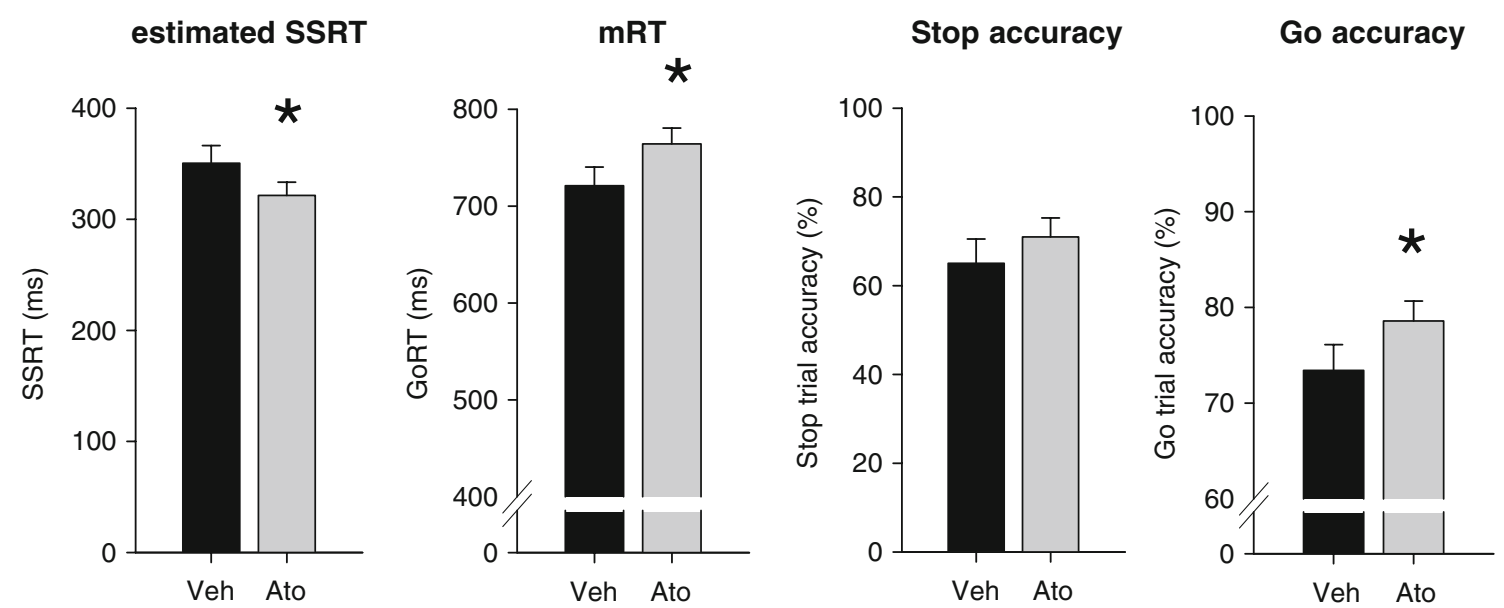

Fig. 3 Effects of atomoxetine $(1 \mathrm{mg} / \mathrm{kg})$ on estimated SSRT, mRT, adjusted stop accuracy, and go accuracy. Atomoxetine improved stop task performance. ${ }^{*} p<0.05$, Drug vs. Veh; $n=24$ ) 


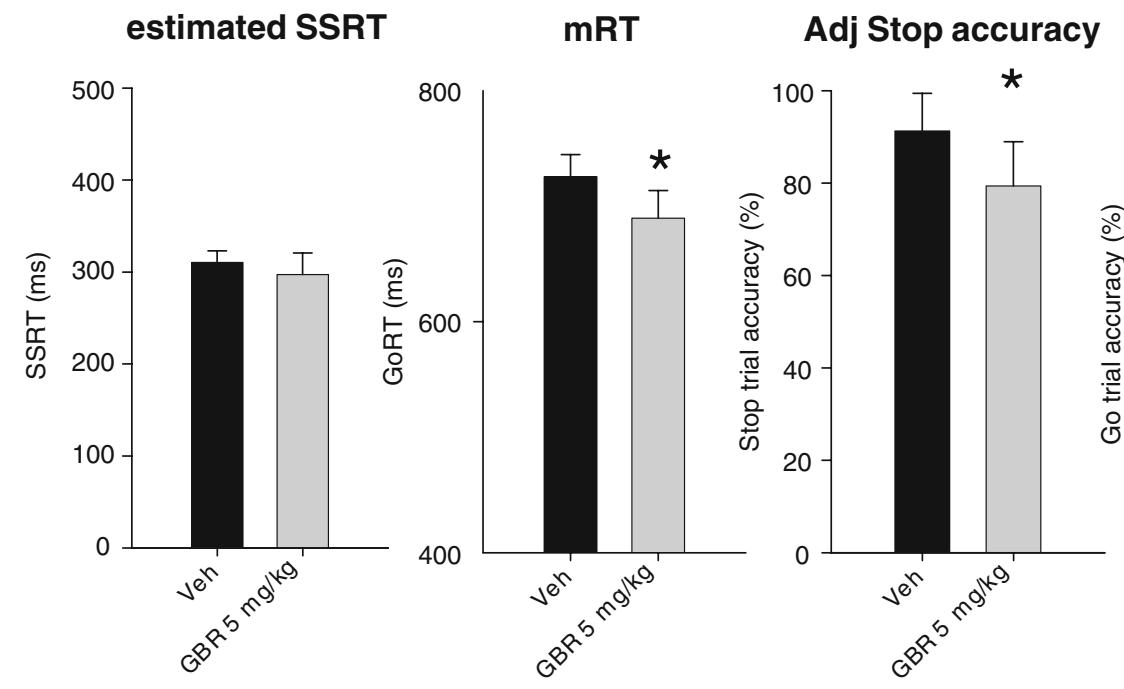

Go accuracy
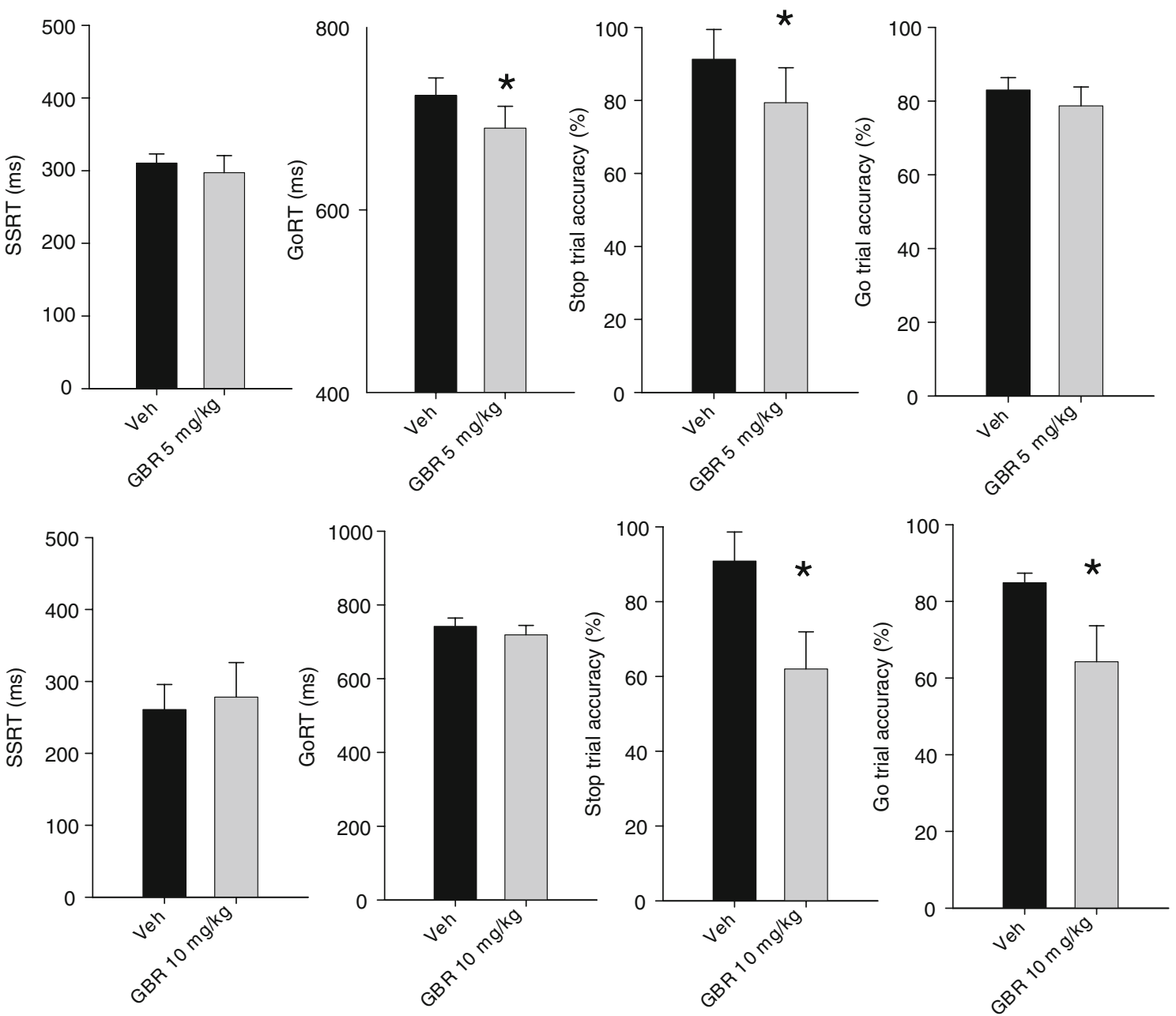

Fig. 4 Effects of higher doses of GBR-12909 administered by i.p. route. Five milligram per kilogram speeded reaction time and impaired stop accuracy (upper panel); $10 \mathrm{mg} / \mathrm{kg}$ impaired both stop and go accuracy with no effects on reaction times (lower panel). * $p<0.05$, Drug vs. Veh; $n=12$ )

Experiment 4: effects of guanfacine administration

Repeated-measures analyses of variance revealed a significant main effect of guanfacine treatment on estimated SSRT (Fig. 5, upper left; $F(4,96)=12.4, p<0.001$ ). Pairwise tests showed that only $0.3 \mathrm{mg} / \mathrm{kg}$ significantly slowed SSRT compared to all other doses.

There was a main effect of guanfacine on mRT (Fig. 5, upper right; $F(4,64)=20.02, p<0.001)$. More specifically, pairwise comparisons showed a slower mRT at higher doses compared to vehicle ( 0 vs. $0.1 \mathrm{mg} / \mathrm{kg}, p<0.02 ; 0$ vs. $0.3 \mathrm{mg} / \mathrm{kg}, p<0.001)$. Guanfacine decreased both adjusted stop and go-trial accuracy (Fig. 5, lower left and right; go accuracy: $F(3,52)=32.2, p<0.001$; adjusted stop accuracy: $F(4,68)=20.4, p<0.001)$. In both cases, only $0.3 \mathrm{mg} / \mathrm{kg}$ was significantly different from vehicle.

\section{Discussion}

We have replicated recent findings of speeding effects of the SNRI atomoxetine on the SSRT measure of behavioral inhibition in humans (Chamberlain et al. 2006) and rats (Robinson et al. 2008). Blocking DA transporter by GBR12909 administration speeded $\mathrm{mRT}$ at the dose of $5 \mathrm{mg} / \mathrm{kg}$, but SERT blockade and guanfacine administration had only minor effects on the general performance.

These results suggest that (a) atomoxetine may exert its effects on SSRT via NA rather than DA reuptake blockade; (b) there may be dissociable effects of central DA and NA mechanisms on hypothetical "go" and "stop" processes, respectively; and (c) blockade of serotonin reuptake over a wide range of doses failed to affect SSRT, congruent with human data (Chamberlain et al. 2006). 

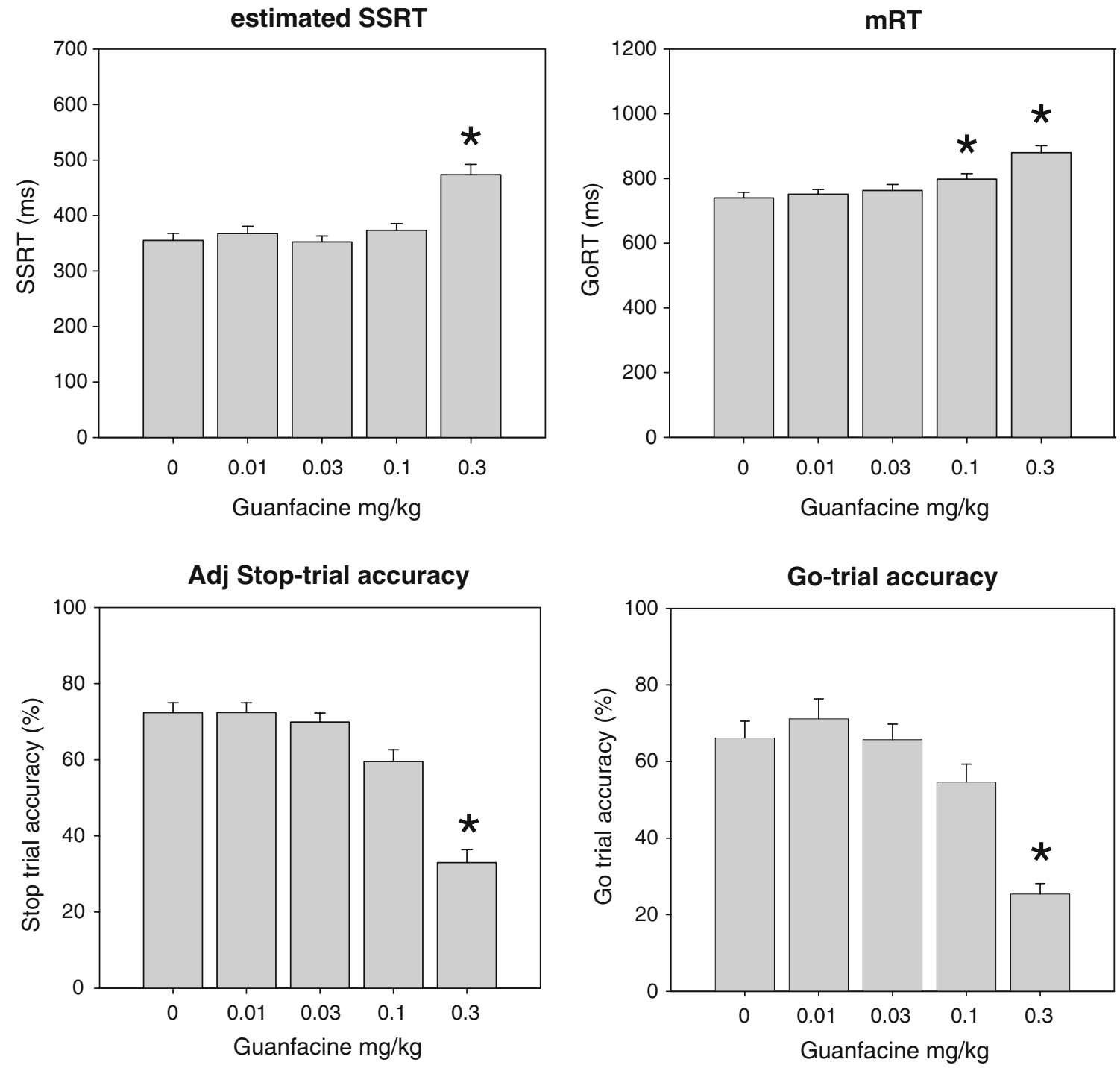

Fig. 5 Effects of guanfacine on estimated SSRT, mRT, and stop and go accuracies. Both reaction times were significantly slowed by the highest dose tested. Go and stop accuracies were markedly decreased at $0.3 \mathrm{mg} / \mathrm{kg}$. ${ }^{*} p<0.001$ vs. vehicle; $n=25$ )

According to the present results, a study by Overtoom et al. (2003), found that administration of the tricyclic antidepressant desipramine speeded SSRTs in children with ADHD. The authors proposed that the beneficial effects might have been caused by the modulation of 5-HT levels by desipramine, which blocks NET like atomoxetine, but also inhibits the 5-HT transporter (Richelson and Pfenning 1984; Rothman et al. 2001), although to a lesser extent. In the present study, blocking 5-HT reuptake by citalopram administration did not improve stopping performance in rats, but NET blockade with atomoxetine did. This result is consistent with what has been found in normal human subjects (Chamberlain et al. 2006) and broadens the range of citalopram doses tested, though revealing significant effects only on secondary performance variables. Go-trial accuracy was decreased and mRT increased with $1 \mathrm{mg} / \mathrm{kg}$ of citalopram, probably because of the mild sedative effects of the drug. Considering the evidence from recent experiments in humans with acute tryptophan depletion (Clark et al. 2005) - which acutely decreases central 5-HT synthesisand global 5-HT depletion in animals (Eagle et al. 2009), that did not affect stopping performance, the present results support the apparent lack of effect of modulating 5-HT transmission on SSRT. Serotonergic neurotransmission has been reported to play a prominent role in a different kind of inhibition, namely, the capacity to withhold over time a response to affectively charged stimuli and to delay gratification (Eagle et al. 2009; Harrison et al. 1997, 1999; Soubrié 1986; Wogar et al. 1993), and thus be more implicated in cognitive - rather than motor-inhibition. Nevertheless, also due to a long-standing link between 5-HT and behavioral inhibition (Evenden 1999), opposite roles of different serotonergic receptors on measures of impulsivity (Carli et al. 2006; Winstanley et al. 2004) and a 
possible association between SSRT and a genetic marker of the human neuronal tryptophan hydroxylase-2 (the ratelimiting enzyme for serotonin biosynthesis; Stoltenberg et al. 2006), 5-HT involvement in stopping deserves further investigation and a study employing subchronic or chronic administration of SSRIs is still lacking.

We recently demonstrated that atomoxetine dosedependently speeds up SSRT and that this effect was not dependent on animals' baseline performance (Robinson et al. 2008). In the present study, we also obtained a significant improvement in go accuracy and a slowing of $\mathrm{mRT}$ at $1 \mathrm{mg} / \mathrm{kg}$. Both effects were present as trends in the study by Robinson et al. (2008), though they did not reach significance. This could be due to the slightly different and more stringent parameters used in this study (i.e., shorter $\mathrm{LH}$ ) or to the fact that more subjects have been used in the present study, which provided greater statistical power. Moreover, a different route of drug administration (i.p. in the previous report vs. s.c. in the present study) could have influenced the drug's absorption rate and distribution. The fact that atomoxetine slowed mRT cannot, of course, explain the speeding effect on SSRT. In fact, even though a slower GoRT can facilitate stopping performance, our procedure takes into account such slowing effects and allows setting delays based on the actual GoRT after drug administration. A longer GoRT is consistent with the increase in alpha-2 inhibitory autoreceptor stimulation in the locus coeruleus as a consequence of heightened NA levels (De Sarro et al. 1987), although, at low doses, it becomes apparent only under very challenging task demands. The beneficial effect on go accuracy is more difficult to explain. One possibility is that atomoxetine makes animals less prone to interrupt the chain of actions required to complete a go response, and thus better at inhibiting premature responses to the food magazine in the middle of the sequence, which, during go-trials, would be likely to cause the rat to exceed the LH and, hence, commit a go-error. On the other hand, atomoxetine could improve the animal's capacity for sustained attention over many trials and thus enable effective application of task contingencies.

GBR-12909 speeded $\mathrm{mRT}$ at $5 \mathrm{mg} / \mathrm{kg}$, decreased go accuracy at $10 \mathrm{mg} / \mathrm{kg}$, and caused a marked decrease in stop accuracy at both doses. The observed increase in the speed of mRT after GBR-12909 administration is consistent with its DA-enhancing effect in DAT-rich subcortical areas like the striatum. In fact, in the prefrontal cortex, DAT distribution is very sparse (Sesack et al. 1998) and most extracellular DA is transported inside the neuron terminals by NET (Carboni et al. 1990). The apparent general disruption of subjects' performance in this study caused by a selective dopaminergic agent like GBR-12909 seems in contrast with the efficacy of psychostimulants in treating
ADHD and speeding SSRT (e.g., Tannock et al. 1989). One possibility is that the doses that caused such effects ( 5 and $10 \mathrm{mg} / \mathrm{kg}$ ) were too high and, hence, at the right end of the "inverted U" shaped curve which is a typical dose-response curve for many behavioral effects elicited by catecholaminergic manipulations (Arnsten and Li 2005; Robbins 2000; Williams and Goldman-Rakic 1995; Zahrt et al. 1997). In the first part of the experiment, we used low doses of GBR12909 delivered by subcutaneous route of administration that did not cause any appreciable effect on stop task variables. The two additional highest doses were then delivered intraperitoneally probably allowing for a more rapid absorption of the drug and a more potent effect on DA release (Pozzi et al. 1994; Tanda et al. 1997) which disrupted rats' performance. More likely, differences in the mechanisms of action between commonly prescribed ADHD drugs and GBR-12909 can explain the results obtained. Compared to amphetamine and methylphenidate, GBR-12909 has the lowest affinity for NET and the highest for DAT (Richelson and Pfenning 1984; Rothman et al. 2001). Consequently, GBR-12909 causes only less than a twofold increase in the extracellular concentration of DA in medial PFC (Mazei et al. 2002; Tanda et al. 1997) due to the paucity of DAT sites in this area (Sesack et al. 1998; Soucy et al. 1997), but dramatically increases DA-like other psychostimulants - in DAT-rich subcortical areas (Tanda et al. 1997). On the contrary, blocking NET with atomoxetine increases DA-as well as NE-in mPFC, but not in the striatum (Bymaster et al. 2002). Half-way between selective NET and DAT blockers, amphetamine and methylphenidate evoke a larger increase of extracellular DA in PFC than in striatal areas (Bymaster et al. 2002; Tanda et al. 1997).

Guanfacine, a selective alpha-2 adrenergic receptor agonist, has been proposed as a potential treatment for ADHD and as a useful alternative to psychostimulant medication (Scahill et al. 2001; Taylor and Russo 2001). Guanfacine is known to have beneficial effects on working memory and attentional functions (Arnsten and Li 2005), but imbalances in dopaminergic or noradrenergic function may mediate the efficacy of low doses of this drug (Arnsten et al. 1988; Franowicz and Arnsten 1998). For example, guanfacine failed to improve behavioral performance in the absence of any induced or pre-existing impairment (Birnbaum et al. 2000). On the other hand, in other studies, alpha-2 adrenoceptor agonist administration had deleterious consequences on attention (Smith and Nutt 1996) and target detection (Coull et al. 2004). Guanfacine, like the other drugs used in the present report, had no differential effects in fast or slow stoppers (separated by median split of the sample (see Eagle et al. 2007); data not shown) suggesting that the effects found are similar in both "normal" and behavioral impaired animals. 
In our study, guanfacine significantly prolonged SSRT and mRT and decreased both stop and go accuracy at the highest dose tested $(0.3 \mathrm{mg} / \mathrm{kg})$. These effects are probably caused by a general slowing of performance-likely caused by feedback inhibition of noradrenaline release by presynaptic alpha 2-receptors stimulation (De Sarro et al. 1987; Engberg and Eriksson 1991) — and not by a decrease in attentional capacity. In the rat version of the task, the time available to press the right lever is limited and this causes the slowest part of the GoRT distribution to be truncated. Stop accuracy is then decreased because it has to be adjusted for high levels of omissions on go-trials. A recent study from this laboratory conducted in humans failed to find improvements by guanfacine on executive and memory functions in healthy subjects (Müller et al. 2005). More specifically, in the stop-signal paradigm, $1 \mathrm{mg}$ of guanfacine slowed mRT but not SSRT (similarly to the effects of $0.1 \mathrm{mg} / \mathrm{kg}$ in the present study), and this effect was unlikely to have been caused by more frequent lapses of attention. These results resemble very closely the effects found in this study on rats. In fact, guanfacine did not affect the GoRT variance even at the highest dose used $(0.3 \mathrm{mg} / \mathrm{kg}$; data not shown), this measure being known to reflect fluctuations in attentional processing (Castellanos and Tannock 2002).

Available data from human subjects are insufficient for us to draw clear conclusions about the relative roles of DA and NA in stopping performance. According to the present results, we can exclude an increase in subcortical extracellular dopamine from being either sufficient or necessary in ameliorating stopping performance because GBR-12909 failed to speed SSRT or to increase stop accuracy, while atomoxetine speeded SSRT. Overtoom et al. (2003) found that L-Dopa administration in ADHD subjects did not alter performance in the stop-signal task and methylphenidate speeded reaction times during go-trials only. Apart from this latter study, where methylphenidate doses were probably too low to speed SSRT, the beneficial effects of methylphenidate and other psychostimulant drugs on stopping performance often appear to critically depend on neurobiological and behavioral baseline levels (Boonstra et al. 2005; de Wit et al. 2000; Eagle and Robbins 2003; Eagle et al. 2007; Feola et al. 2000; Vaidya et al. 1998; Volkow et al. 1997, 2004).

As a consequence of the paucity of DAT in the PFC, SNRIs like atomoxetine, despite their high selectivity for the NA transporter, cause an increase of extracellular DA as well as NA there (Bymaster et al. 2002; Carboni et al. 1990; Gresch et al. 1995). The lower abuse viability of SNRI and increased safety in the treatment of ADHD patients compared to commonly used psychostimulant drugs has been attributed to their relative lack of effect in the DA-rich striatum. On the other hand, atomoxetine may help to exert a prefrontal inhibitory influence on subcortical regions, including the striatum, via its effects on cortical catecholamines (Deutch 1992; Zametkin and Rapoport 1987).

In summary, the present data concur with previous results in humans and rats (e.g., Chamberlain et al. 2006; Eagle et al. 2007; Overtoom et al. 2003; Robinson et al. 2008) suggesting a dissociation between the neurochemical modulation of stopping performance by noradrenaline and the control of go processes by dopaminergic neurotransmission.

Acknowledgments This research was supported by a Wellcome Trust Programme Grant $(076274 / \mathrm{z} / 04 / \mathrm{z})$ and completed within the Cambridge University Behavioural and Clinical Neuroscience Institute, supported by a joint award from the Medical Research Council (MRC) and Wellcome Trust. AB was supported by an MRC studentship and by Pfizer Inc. ESJR holds an RCUK Academic Fellowship supported by the British Pharmacological Society Integrative Pharmacology Fund. The author would like to thank Barry J. Everitt and Anne W. Schmidt for advice and David E. Theobald for skilful assistance.

\section{References}

Arnsten AF, Li BM (2005) Neurobiology of executive functions: catecholamine influences on prefrontal cortical functions. Biol Psychiatry 57:1377-1384

Arnsten AF, Cai JX, Goldman-Rakic PS (1988) The alpha-2 adrenergic agonist guanfacine improves memory in aged monkeys without sedative or hypotensive side effects: evidence for alpha-2 receptor subtypes. J Neurosci 8:4287-4298

Aron AR, Fletcher PC, Bullmore ET, Sahakian BJ, Robbins TW (2003) Stop-signal inhibition disrupted by damage to right inferior frontal gyrus in humans. Nat Neurosci 6:115-116

Barkley RA (1997) Behavioral inhibition, sustained attention, and executive functions: constructing a unifying theory of ADHD. Psychol Bull 121:65-94

Birnbaum SG, Podell DM, Arnsten AF (2000) Noradrenergic alpha-2 receptor agonists reverse working memory deficits induced by the anxiogenic drug, FG7142, in rats. Pharmacol Biochem Behav 67:397-403

Boonstra AM, Kooij JJ, Oosterlaan J, Sergeant JA, Buitelaar JK (2005) Does methylphenidate improve inhibition and other cognitive abilities in adults with childhood-onset ADHD? J Clin Exp Neuropsychol 27:278-298

Bymaster FP, Katner JS, Nelson DL, Hemrick-Luecke SK, Threlkeld PG, Heiligenstein JH, Morin SM, Gehlert DR, Perry KW (2002) Atomoxetine increases extracellular levels of norepinephrine and dopamine in prefrontal cortex of rat: a potential mechanism for efficacy in attention deficit/hyperactivity disorder. Neuropsychopharmacology 27:699-711

Carboni E, Tanda GL, Frau R, Di Chiara G (1990) Blockade of the noradrenaline carrier increases extracellular dopamine concentrations in the prefrontal cortex: evidence that dopamine is taken up in vivo by noradrenergic terminals. J Neurochem 55:10671070

Cardinal RN, Aitken MRF (2001) Whisker, version 2.2, computer software.

Carli M, Baviera M, Invernizzi RW, Balducci C (2006) Dissociable contribution of 5-HT1A and 5-HT2A receptors in the medial prefrontal cortex to different aspects of executive control such as 
impulsivity and compulsive perseveration in rats. Neuropsychopharmacology 31:757-767

Castellanos FX, Tannock R (2002) Neuroscience of attention-deficit/ hyperactivity disorder: the search for endophenotypes. Nat Rev Neurosci 3:617-628

Chamberlain SR, Müller U, Blackwell AD, Clark L, Robbins TW, Sahakian BJ (2006) Neurochemical modulation of response inhibition and probabilistic learning in humans. Science 311:861-863

Clark L, Roiser JP, Cools R, Rubinsztein DC, Sahakian BJ, Robbins TW (2005) Stop signal response inhibition is not modulated by tryptophan depletion or the serotonin transporter polymorphism in healthy volunteers: implications for the 5-HT theory of impulsivity. Psychopharmacology (Berl) 182:570-578

Coull JT, Jones ME, Egan TD, Frith CD, Maze M (2004) Attentional effects of noradrenaline vary with arousal level: selective activation of thalamic pulvinar in humans. Neuroimage 22:315322

De Sarro GB, Ascioti C, Froio F, Libri V, Nistico G (1987) Evidence that locus coeruleus is the site where clonidine and drugs acting at alpha 1- and alpha 2-adrenoceptors affect sleep and arousal mechanisms. Br J Pharmacol 90:675-685

de Wit H, Crean J, Richards JB (2000) Effects of d-amphetamine and ethanol on a measure of behavioral inhibition in humans. Behav Neurosci 114:830-837

Deutch AY (1992) The regulation of subcortical dopamine systems by the prefrontal cortex: interactions of central dopamine systems and the pathogenesis of schizophrenia. J Neural Transm Suppl 36:61-89

Devoto P, Flore G, Pira L, Longu G, Gessa GL (2004) Alpha2adrenoceptor mediated co-release of dopamine and noradrenaline from noradrenergic neurons in the cerebral cortex. J Neurochem 88:1003-1009

Eagle DM, Robbins TW (2003) Inhibitory control in rats performing a stop-signal reaction-time task: effects of lesions of the medial striatum and d-amphetamine. Behav Neurosci 117:1302-1317

Eagle DM, Tufft MR, Goodchild HL, Robbins TW (2007) Differential effects of modafinil and methylphenidate on stop-signal reaction time task performance in the rat, and interactions with the dopamine receptor antagonist cis-flupenthixol. Psychopharmacology (Berl) 192:193-206

Eagle DM, Baunez C, Hutcheson DM, Lehmann O, Shah AP, Robbins TW (2008) Stop-signal reaction-time task performance: role of prefrontal cortex and subthalamic nucleus. Cereb Cortex 18:178-188

Eagle DM, Lehmann O, Theobald DEH, Pena Y, Zakaria R, Ghosh R, Dalley JW, Robbins TW (2009) Serotonin depletion impairs waiting but not stop-signal reaction time in rats: implications for theories of the role of 5-HT in behavioral inhibition. Neuropsychopharmacology 34(5):1311-1321

Easton N, Shah YB, Marshall FH, Fone KC, Marsden CA (2006) Guanfacine produces differential effects in frontal cortex compared with striatum: assessed by phMRI BOLD contrast. Psychopharmacology (Berl) 189:369-385

Engberg G, Eriksson E (1991) Effects of alpha 2-adrenoceptor agonists on locus coeruleus firing rate and brain noradrenaline turnover in N-ethoxycarbonyl-2-ethoxy-1, 2-dihydroquinoline (EEDQ)-treated rats. Naunyn Schmiedebergs Arch Pharmacol 343:472-477

Evenden JL (1999) Varieties of impulsivity. Psychopharmacology (Berl) 146:348-361

Feola TW, de Wit H, Richards JB (2000) Effects of d-amphetamine and alcohol on a measure of behavioral inhibition in rats. Behav Neurosci 114:838-848

Franowicz JS, Arnsten AF (1998) The alpha-2a noradrenergic agonist, guanfacine, improves delayed response performance in young adult rhesus monkeys. Psychopharmacology (Berl) 136:8-14
Gresch PJ, Sved AF, Zigmond MJ, Finlay JM (1995) Local influence of endogenous norepinephrine on extracellular dopamine in rat medial prefrontal cortex. J Neurochem 65:111-116

Groman SM, James AS, Jentsch JD (2007) Poor response inhibition: at the nexus between substance abuse and attention deficit/ hyperactivity disorder. Neurosci Biobehav Rev. doi:10.1016/j. neubiorev.2008.08.008

Harrison AA, Everitt BJ, Robbins TW (1997) Central 5-HT depletion enhances impulsive responding without affecting the accuracy of attentional performance: interactions with dopaminergic mechanisms. Psychopharmacology (Berl) 133:329-342

Harrison AA, Everitt BJ, Robbins TW (1999) Central serotonin depletion impairs both the acquisition and performance of a symmetrically reinforced go/no-go conditional visual discrimination. Behav Brain Res 100:99-112

Heal DJ, Smith SL, Kulkarni RS, Rowley HL (2008) New perspectives from microdialysis studies in freely-moving, spontaneously hypertensive rats on the pharmacology of drugs for the treatment of ADHD. Pharmacol Biochem Behav 90(2):184-197

Horner WE, Johnson DE, Schmidt AW, Rollema H (2007) Methylphenidate and atomoxetine increase histamine release in rat prefrontal cortex. Eur J Pharmacol 558:96-97

Howell DC (1997) Statistical methods for psychology, 4th edn. Duxbury, Belmont

Iversen L (2006) Neurotransmitter transporters and their impact on the development of psychopharmacology. Br J Pharmacol 147(Suppl 1):S82-S88

Logan GD (1994) On the ability to inhibit thought and action. A users' guide to the stop signal paradigm. In: Dagenbach D, Carr $\mathrm{TH}$ (eds) Inhibitory processes in attention memory and language. Academic, San Diego, CA, pp 189-236

Logan GD, Cowan WB, Davis KA (1984) On the ability to inhibit simple and choice reaction time responses: a model and a method. J Exp Psychol Hum Percept Perform 10:276-291

Masson J, Sagne C, Hamon M, El Mestikawy S (1999) Neurotransmitter transporters in the central nervous system. Pharmacol Rev $51: 439-464$

Mazei MS, Pluto CP, Kirkbride B, Pehek EA (2002) Effects of catecholamine uptake blockers in the caudate-putamen and subregions of the medial prefrontal cortex of the rat. Brain Res 936:58-67

Moeller FG, Barratt ES, Dougherty DM, Schmitz JM, Swann AC (2001) Psychiatric aspects of impulsivity. Am J Psychiatry 158:1783-1793

Müller U, Clark L, Lam ML, Moore RM, Murphy CL, Richmond NK, Sandhu RS, Wilkins IA, Menon DK, Sahakian BJ, Robbins TW (2005) Lack of effects of guanfacine on executive and memory functions in healthy male volunteers. Psychopharmacology (Berl) 182:205-213

Oosterlaan J, Logan GD, Sergeant JA (1998) Response inhibition in $\mathrm{AD} / \mathrm{HD}, \mathrm{CD}$, comorbid $\mathrm{AD} / \mathrm{HD}+\mathrm{CD}$, anxious, and control children: a meta-analysis of studies with the stop task. J Child Psychol Psychiatry 39:411-425

Overtoom CC, Verbaten MN, Kemner C, Kenemans JL, van Engeland $\mathrm{H}$, Buitelaar JK, van der Molen MW, van der Gugten J, Westenberg H, Maes RA, Koelega HS (2003) Effects of methylphenidate, desipramine, and L-dopa on attention and inhibition in children with attention deficit hyperactivity disorder. Behav Brain Res 145:7-15

Pozzi L, Invernizzi R, Cervo L, Vallebuona F, Samanin R (1994) Evidence that extracellular concentrations of dopamine are regulated by noradrenergic neurons in the frontal cortex of rats. J Neurochem 63:195-200

Richelson E, Pfenning M (1984) Blockade by antidepressants and related compounds of biogenic amine uptake into rat brain synaptosomes: most antidepressants selectively block norepinephrine uptake. Eur J Pharmacol 104:277-286 
Robbins TW (2000) Chemical neuromodulation of frontal-executive functions in humans and other animals. Exp Brain Res 133:130 138

Robbins TW (2007) Shifting and stopping: fronto-striatal substrates, neurochemical modulation and clinical implications. Philos Trans R Soc Lond B Biol Sci 362:917-932

Robinson ES, Eagle DM, Mar AC, Bari A, Banerjee G, Jiang X, Dalley JW, Robbins TW (2008) Similar effects of the selective noradrenaline reuptake inhibitor atomoxetine on three distinct forms of impulsivity in the rat. Neuropsychopharmacology 33:1028-1037

Rothman RB, Baumann MH, Dersch CM, Romero DV, Rice KC, Carroll FI, Partilla JS (2001) Amphetamine-type central nervous system stimulants release norepinephrine more potently than they release dopamine and serotonin. Synapse 39:32-41

Rubia K (2002) The dynamic approach to neurodevelopmental psychiatric disorders: use of fMRI combined with neuropsychology to elucidate the dynamics of psychiatric disorders, exemplified in ADHD and schizophrenia. Behav Brain Res 130:47-56

Rubia K, Oosterlaan J, Sergeant JA, Brandeis D, v Leeuwen T (1998) Inhibitory dysfunction in hyperactive boys. Behav Brain Res 94:25-32

Sagvolden T (2006) The alpha-2A adrenoceptor agonist guanfacine improves sustained attention and reduces overactivity and impulsiveness in an animal model of attention-deficit/hyperactivity disorder (ADHD). Behav Brain Funct 2:41

Scahill L, Chappell PB, Kim YS, Schultz RT, Katsovich L, Shepherd E, Arnsten AF, Cohen DJ, Leckman JF (2001) A placebocontrolled study of guanfacine in the treatment of children with tic disorders and attention deficit hyperactivity disorder. Am J Psychiatry 158:1067-1074

Semrud-Clikeman M, Steingard RJ, Filipek P, Biederman J, Bekken K, Renshaw PF (2000) Using MRI to examine brain-behavior relationships in males with attention deficit disorder with hyperactivity. J Am Acad Child Adolesc Psychiatry 39:477-484

Sesack SR, Hawrylak VA, Guido MA, Levey AI (1998) Cellular and subcellular localization of the dopamine transporter in rat cortex. Adv Pharmacol 42:171-174

Smith A, Nutt D (1996) Noradrenaline and attention lapses. Nature 380:291

Soubrié P (1986) Serotonergic neurons and behavior. J Pharmacol 17:107-112

Soucy JP, Mrini A, Lafaille F, Doucet G, Descarries L (1997) Comparative evaluation of [3H]WIN 35428 and [3H]GBR 12935 as markers of dopamine innervation density in brain. Synapse 25:163-175

Stoltenberg SF, Glass JM, Chermack ST, Flynn HA, Li S, Weston ME, Burmeister M (2006) Possible association between response inhibition and a variant in the brain-expressed tryptophan hydroxylase-2 gene. Psychiatr Genet 16:35-38

Tanda G, Pontieri FE, Frau R, Di Chiara G (1997) Contribution of blockade of the noradrenaline carrier to the increase of extracellular dopamine in the rat prefrontal cortex by amphetamine and cocaine. Eur J Neurosci 9:2077-2085

Tannock R, Schachar RJ, Carr RP, Chajczyk D, Logan GD (1989) Effects of methylphenidate on inhibitory control in hyperactive children. J Abnorm Child Psychol 17:473-491

Taylor FB, Russo J (2001) Comparing guanfacine and dextroamphetamine for the treatment of adult attention-deficit/hyperactivity disorder. J Clin Psychopharmacol 21:223-228

Tzavara ET, Bymaster FP, Overshiner CD, Davis RJ, Perry KW, Wolff M, McKinzie DL, Witkin JM, Nomikos GG (2006) Procholinergic and memory enhancing properties of the selective norepinephrine uptake inhibitor atomoxetine. Mol Psychiatry 11:187-195

Vaidya CJ, Austin G, Kirkorian G, Ridlehuber HW, Desmond JE, Glover GH, Gabrieli JD (1998) Selective effects of methylphenidate in attention deficit hyperactivity disorder: a functional magnetic resonance study. Proc Natl Acad Sci U S A 95:1449414499

Volkow ND, Wang GJ, Fowler JS, Logan J, Angrist B, Hitzemann R, Lieberman J, Pappas N (1997) Effects of methylphenidate on regional brain glucose metabolism in humans: relationship to dopamine D2 receptors. Am J Psychiatry 154:50-55

Volkow ND, Wang GJ, Fowler JS, Telang F, Maynard L, Logan J, Gatley SJ, Pappas N, Wong C, Vaska P, Zhu W, Swanson JM (2004) Evidence that methylphenidate enhances the saliency of a mathematical task by increasing dopamine in the human brain. Am J Psychiatry 161:1173-1180

Williams GV, Goldman-Rakic PS (1995) Modulation of memory fields by dopamine D1 receptors in prefrontal cortex. Nature 376:572-575

Winstanley CA, Theobald DE, Dalley JW, Glennon JC, Robbins TW (2004) 5-HT2A and 5-HT2C receptor antagonists have opposing effects on a measure of impulsivity: interactions with global 5HT depletion. Psychopharmacology (Berl) 176:376-385

Wogar MA, Bradshaw CM, Szabadi E (1993) Effect of lesions of the ascending 5-hydroxytryptaminergic pathways on choice between delayed reinforcers. Psychopharmacology (Berl) 111:239-243

Zahrt J, Taylor JR, Mathew RG, Arnsten AF (1997) Supranormal stimulation of D1 dopamine receptors in the rodent prefrontal cortex impairs spatial working memory performance. J Neurosci $17: 8528-8535$

Zametkin AJ, Rapoport JL (1987) Neurobiology of attention deficit disorder with hyperactivity: where have we come in 50 years? J Am Acad Child Adolesc Psychiatry 26:676-686 